\section{FUNCTIONAL GASTROINTESTINAL DISORDERS IN A TERTIARY OUTPATIENT SETTING - A THREE-YEAR PERIOD OUTCOME}

${ }^{1}$ Katarina Milošić ${ }^{*}$ 2,3 Mirna Natalija Aničić, ${ }^{3}$ Lana Omerza, ${ }^{2,3}$ Irena Senečíc-Čala, 2,3Jurica Vuković, 2,3 Duška Tješić-Drinković. 'Department of Emergency Medicine of KrapinaZagorje County, Dr. Mirka Crkvenca 1, 49000 Krapina; ${ }^{2}$ Zagreb School of Medicine, Šalata 3, 10000 Zagreb; ${ }^{3}$ UHC Zagreb, Department of Paediatrics, Kišpatićeva 12, 10000 Zagreb

10.1136/archdischild-2021-europaediatrics.251

Goal The aim of this study was to evaluate children with functional gastrointestinal disorders (FGIDs) seen by the paediatric gastroenterologist (PG) and their outcome during a threeyear period.

Method The study included children with FGIDs who visited the PG at the UHC Zagreb from January 1st 2017 to December 31st $2017(\mathrm{~N}=328)$. This retrospective cohort was followed until December 31st 2019, data was extracted retrospectively from clinical records and their outcomes were assessed. Descriptive statistics and McNemar's test were used, statistical significance was determined as $\mathrm{p}<0.05$.

Results About 2/3 of outpatients (222/328) dropped out during the year 2017. The leading diagnosis was functional constipation $(90 / 222$ or $40.5 \%)$ and more than half of these patients were younger than 11 years (106/222).

Most drop-outs visited the PG only once in 2017 (134/ 222), but for 33 patients this was a control visit, meaning that 101/222 (45.5\%) needed no further subspecialist's followups after the index visit. During the year 2018 the number of patients who dropped out was $63 / 106(59.4 \%)$ or $1 / 5$ of the initial group. Finally, $43 / 328$ or $13.1 \%$ of children from the initial cohort were still supervised by PG during the year 2019. About half of them had functional constipation (20/43), followed by irritable bowel syndrome (IBS) (12/43) and other functional abdominal pain disorders. Majority of patients were in the adolescent group (28/43). Further, we assessed the severity of symptoms at their last appointment in a subgroup of 227 patients who had one or more check-ups in the threeyear period. About half of the patients claimed some improvement (115/227 patients), while $1 / 5$ stated they were symptomfree (49/227). About $1 / 4$ of children reported no change in the severity of symptoms (58/227). The vast majority of patients were correctly diagnosed with FGID, although the type of FGID changed in $13 / 328$ subjects.

In one patient, however, the diagnosis of IBS was reversed to ulcerative colitis. A significant number of children was included in a child psychologist/psychiatrist treatment $(74 / 328$ or $22.5 \%)$.

Conclusion This survey reveals that almost half of children referred to the PG because of FGID needs only one subspecialist consultation. Less than $15 \%$ of children with FGID have persistent complaints lasting three years and requiring prolonged PG follow-ups. Adolescents tend to have more pronounced symptoms difficult to treat, as they were more prevalent in the subgroup followed to 2019 than in the initial 2017 cohort $(28 / 43$ vs. $143 / 328, \mathrm{p}<0.001)$

\section{EVALUATION OF CHANGES IN NUTRITIONAL STATUS IN RE-HOSPITALIZED CHILDREN}

Gabriela Živković*, Lana Omerza, Mirna Natalija Aničić, Irena Senečić-Čala, Jurica Vuković, Duška Tješić-Drinković. University of Zagreb, Zagreb School of Medicine, Zagreb

10.1136/archdischild-2021-europaediatrics.252
This is a one-year follow-up study looking into the nutritional status and the rate of re-hospitalizations in children at the UHC Zagreb, Dept. of Paediatrics who were first evaluated during the nutritionDay (nDay) in November 2018. The aim is to evaluate the accuracy of STRONGkids questionnaires, subjective assessment within nDay survey and anthropometry in detecting malnutrition and possible relation to number of hospital admissions and disease outcomes (for oncology patients) within a year.

The study included 50 patients (mean age 13.48 years $\pm 3.79,22$ males) whose nutritional status was estimated in November 2018. Additional data were collected after the period of 12 months. Mann-Whitney U, Kruskal Wallis and Wilcoxon signed-ranks tests were applied.

A significantly different BMI was found among subgroups categorized through the nDay survey (without risk, at risk, malnourished patients) and among subgroups assessed by STRONGkids (low, medium and high risk for malnutrition) $(\mathrm{p} 1=0.002, \mathrm{p} 2=0.003$, resp.). Post hoc tests showed that statistical significance could be contributed to differences between groups malnourished patients vs. those not at risk within nDay $(\mathrm{p} 3=0.009)$ and between groups low vs. high and medium vs. high risk defined through STRONGkids questionnaires ( $\mathrm{p} 4=0.020, \mathrm{p} 5=0.004$, resp.). In the one-year follow-up period, 28/50 children were re-hospitalized once or several times.

The number of re-hospitalizations was significantly higher for children classified by STRONGkids to have high risk for malnutrition ( $\mathrm{p} 6=0.002)$, as well as for those categorized as malnourished through the nDay survey $(\mathrm{p} 7=0.024)$. No significant difference in $z$-score BMI values was found between years 2018 and 2019 (Wilcoxon signed-rank test: $p 8=0.086$ ).

Re-hospitalized oncology patients (11) were additionally analysed in respect of their disease status: favourable outcome (disease regression; 8/11) vs.

unfavourable outcome (progression or unchanged state; 3/ 11). No difference was observed in the initial BMI between these groups (Mann-Whitney U test: $\mathrm{p} 9=0.921$ ), and no significant change in their BMI during this period was observed in either group (Wilcoxon signed-rank test: $\mathrm{p} 10=0.161$, $\mathrm{p} 11=0.593$ )

Patients categorized as malnourished both with STRONGkids and nDay survey had significantly lower BMI than the rest of subjects. Our data support the hypothesis that malnourished patients have a higher rate of re-hospitalizations. Within the observational period, re-hospitalized patients neither improved nor worsened their nutritional status according to the BMI z-scores. We were not able to connect the nutritional status of oncology patients with their disease outcome after one year, perhaps due to a rather small sample or short time of follow-up.

\section{REFRACTORY CYCLIC VOMITING SYNDROME IN A CHILD WITH CHIARI MALFORMATION TYPE II}

Ivana Trivić* , Ana Močić Pavić, Vlasta Đuranović, Hrvoje Jednačak, Ivo Barić, Iva Hojsak, Zrinjka Mišak, Sanja Kolaček, Oleg Jadrešin. Referral Centre for Paediatric Gastroenterology and Nutrition, Children's Hospital Zagreb

10.1136/archdischild-2021-europaediatrics.253

Introduction Cyclic vomiting syndrome (CVS) is a chronic disorder characterized by recurrent episodic attacks of intense 